\title{
Quantum cores of optical phase singularities
}

\section{V Berry and M R Dennis}

H H Wills Physics Laboratory, University of Bristol, Tyndall Avenue, Bristol BS8 1TL, UK

Received 5 September 2003, accepted for publication 8 October 2003

Published 19 April 2004

Online at stacks.iop.org/JOptA/6/S178

DOI: $10.1088 / 1464-4258 / 6 / 5 / 006$

\begin{abstract}
The nodal line singularities (optical vortices) of classical scalar optics are smoothed in quantum optics, because of spontaneous emission into unoccupied modes. The radius of the 'quantum core' surrounding each classical singularity is proportional to $\sqrt{\hbar}$. A trapped excited atom, steered into a nodal line of the classical field, is a possible detector for the effect. Analogous phenomena are anticipated for other waves, for example sound, where the silence at a nodal line is disturbed by pressure fluctuations of the fluid molecules.
\end{abstract}

Keywords: phase singularity, optical vortex, quantum optics, spontaneous emission, decoherence

\begin{abstract}
The geometric structures studied in singular optics [1] are perhaps the most interesting objects predicted by the theories of light. But in a sense they are nonexistent, because each singularity disappears at a deeper level of description. The same is true of other (perhaps all) singularities in physics. Thus, the caustic singularities of geometrical optics disappear in physical (i.e. scalar) optics, being softened by the phase effects introduced by wave physics and replaced by diffraction catastrophes [1-3]. Scalar optics has its own singularities, in the form of nodal lines in space (that is, phase singularities, or optical vortices), and these in turn disappear in polarization (i.e. vector wave) optics, whose singularities are loci of purely linear or purely circular polarization [1, 4].

Our aim here is to extend this process of dissolving singularities to a further level, by exploring an earlier suggestion $[5,6]$ that the quantum vacuum destroys the perfect zero of a classical light field at a phase singularity, replacing it with a quantum core of finite radius. We will calculate this radius. Thus the dark light of a phase singularity can be regarded as a window, opening to our view the faint glimmering of the quantum vacuum. The classical nodal singularity results from perfect destructive interference [7,8], so its quantum smoothing can be regarded as a fundamental decoherence effect. (Smoothing can also arise from classical decoherence, as exhibited in the colours of vortices created with white light $[9,10]$ - a less fundamental effect.)
\end{abstract}

Consider for simplicity a large volume $V$, supporting normalized modes $u_{n}(\mathbf{r})$, here conveniently represented as complex scalar waves (an appropriate description if the field is paraxial and linearly polarized, for example). We are interested in the mode $n=N$, with frequency $\omega_{N}$, and wavelength $\lambda=2 \pi c / \omega_{N}$, that possesses a nodal line passing through the origin and contains classical light, that is, many photons (e.g. in a coherent state). We want to understand how observations of the light in the 'vacuum' near $\mathbf{r}=0$ are affected by the unoccupied modes $n \neq N$, with frequencies degenerate with, or near, $\omega_{N}$, that do not possess nodal lines near $\mathbf{r}=0$. To probe the light on the sub-wavelength scale near a vortex, we choose a single excited atom that can decay with frequency close to $\omega_{N}$, that is, a 'quantum counter' (which could be implemented by a three-level atom) [11, 12].

Light emitted by the atom will be dominated by stimulated emission from $u_{N}$, except near $\mathbf{r}=0$ where spontaneous emission into the unoccupied modes will be important. The intensity (energy density) $I$ (r) of this light can be calculated from the expectation value of the antinormally ordered operator product $\hat{E}(\mathbf{r}) \hat{E}^{\dagger}(\mathbf{r})$, where

$$
\hat{E}(\mathbf{r})=\sum_{n} \hat{a}_{n} u_{n}(\mathbf{r})
$$

is the electric field operator, involving the photon mode annihilation operators $\hat{a}_{n}$. The result is

$$
I(\mathbf{r})=I_{N}(\mathbf{r})+\hbar \omega \sum^{\prime}\left|u_{n}(\mathbf{r})\right|^{2},
$$

whose ingredients will now be described. 
Quantum cores of optical phase singularities
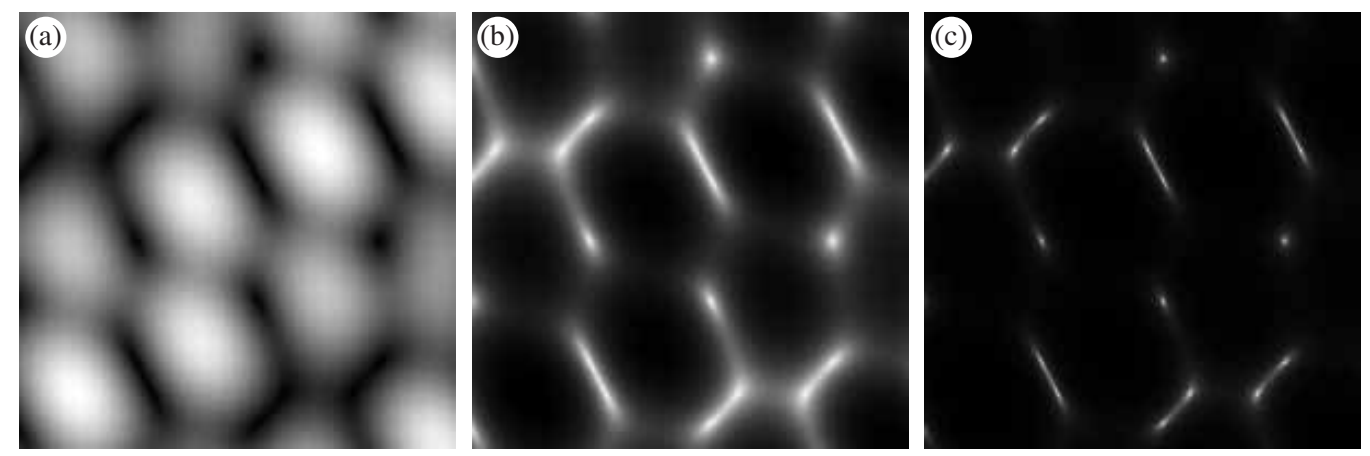

Figure 1. (a) Density plot of modulus $\sqrt{ } I_{N}(r)$ of a superposition of four degenerate plane waves, with mean value $\left\langle I_{N}(\mathbf{r})\right\rangle=1$, showing nodal points (where the nodal lines pierce the plane) as dark spots; the plot is two wavelengths square. (b) Plot of $\rho_{N}(r)$ (equation (9)), for $K=1 / 5$; (c) as (b), with $K=1 / 300$; the white regions are the quantum cores.

The first term $I_{N}(\mathbf{r})$ is the intensity of the classical field, given by the expectation value of the energy density of the photons in the mode $N$, where the light field has state $\left|\phi_{N}\right\rangle$, namely

$$
\begin{aligned}
I_{N}(\mathbf{r}) & =\hbar \omega\left\langle\phi_{N}\left|\hat{a}_{N}^{\dagger} \hat{a}_{N}\right| \phi_{N}\right\rangle\left|u_{N}(\mathbf{r})\right|^{2} \\
& \approx \hbar \omega\left\langle\phi_{N}\left|\hat{a}_{N} \hat{a}_{N}^{\dagger}\right| \phi_{N}\right\rangle\left|u_{N}(\mathbf{r})\right|^{2}
\end{aligned}
$$

(the small difference between the two expressions, representing spontaneous emission involving the state $N$, is irrelevant near a vortex because of the factor $\left|u_{N}\right|^{2}$ ).

The second term in (2) represents the contribution from spontaneous emission, with the prime denoting restriction of the summation to those states $n \neq N$ for which $\left|\omega_{N}-\omega_{n}\right|<$ $\Delta \omega$, where $\Delta \omega$ is the frequency range into which the atom can decay, that is, the linewidth (proportional to Einstein's $A$ coefficient). For large $V$, the density of such states is given by the Weyl rule, and the average value of $\left|u_{n}\right|^{2}$ is $1 / \sqrt{ } V$, so

$$
\sum^{\prime}\left|u_{n}(\mathbf{r})\right|^{2} \approx \frac{\omega^{2} \Delta \omega}{2 \pi^{2} c^{3}}
$$

Consider now the neighbourhood of the optical vortex, where, with the vortex along $\mathrm{O} z$ and $\mathbf{R}=$ $\{x, y\}$, and approximating the usually elliptical vortex intensity contours [13] by circles,

$$
I_{N}(\mathbf{r}) \approx C R^{2}
$$

with $C$ proportional to $\nabla_{\mathbf{R}}^{2} I_{N}(0)$. The quantum core can now be defined as the region within which the 'spontaneous' term (4) (now representing decay into a continuum of unoccupied states) dominates the 'stimulated' term (3). Its radius $R_{\mathrm{Q}}$ is therefore

$$
R_{\mathrm{Q}} \approx \sqrt{\frac{\hbar \omega^{3} \Delta \omega}{2 \pi^{2} c^{3} C}} .
$$

Note that $V$ does not appear in this formula, which therefore applies to unconfined light. The presence of $\Delta \omega$ indicates that the quantum core depends not only on the electromagnetic field but also on the atom employed to detect it.

To estimate $R_{\mathrm{Q}}$, we consider the simplest singular Laguerre-Gauss beam with waist radius $R_{0}$ (that is, intensity profile $\sim R^{2} \exp \left(-R^{2} / R_{0}^{2}\right)$ ) and power $p$. Integration across the beam gives the constant $C$ in (5) as

$$
C=\frac{p}{\pi c R_{0}^{4}} .
$$

Thus the core radius is

$$
R_{\mathrm{Q}}=2 \pi R_{0}^{2} \sqrt{\frac{\hbar c \Delta \omega}{p \lambda^{3}}} .
$$

For a $5 \mathrm{~mW} \mathrm{He}-\mathrm{Ne}$ laser light in a beam with waist radius $100 \mu \mathrm{m}$, detected by a hydrogenic atom with minimum linewidth for spontaneous emission into the visible [14, 15], that is, $\Delta \omega \sim 5 \times 10^{6} \mathrm{~s}^{-1},(8)$ gives $R_{\mathrm{Q}} \sim 0.01 R_{0}$.

The result (6) can be regarded as combining three types of singularity. First, there is the phase singularity that is being smoothed. Second, there is the singular semiclassical (small $\hbar)$ limit, in which the core emerges as a distinct structure (we are here referring to the explicit $\hbar$ dependence associated with the quantization of the light field; of course $\hbar$ also occurs in expressions for $\Delta \omega$, and $\omega$ itself, regarded as the result of transition between atomic energy levels). And third, there is the spectral singularity associated with the degeneracy (or near-degeneracy) of the modes near $N$, into which the atom can decay spontaneously.

In another sense (6) is disappointing, because it shows that the quantum smoothing of a classical-optics singularity appears to possess far less richness than, say, the diffraction catastrophe smoothing of geometrical caustics. The core in (2) merely reflects the inert addition of a constant vacuum term, $K$ say, to the classical intensity. This can however be presented as a localized quantum correction by the contrivance of plotting the amplitude shift

$$
\rho_{N}(\mathbf{r})=\sqrt{I_{N}(\mathbf{r})+K}-\sqrt{I_{N}(\mathbf{r})},
$$

which possesses maxima at each phase singularity and is small elsewhere (figure 1). Nevertheless, the core is a fundamental quantum effect, and it would be interesting to carry out an experiment to detect it, perhaps using the Laguerre-Gauss vortex beam in the estimate above.

The spontaneously emitted light renders the phase $\chi$ of the radiation $\psi=\rho \exp (\mathrm{i} \chi)$ uncertain in the core. At the position $x=r \cos \phi, x=r \sin \phi$, the phase probability distribution can be calculated to be (in dimensionless units)

$$
\begin{aligned}
& P(\chi ; r, \phi)=\frac{1}{2 \pi}\left[\exp \left(-\frac{1}{2} r^{2}\right)\right. \\
& \quad+r \cos (\chi-\phi) \exp \left(-\frac{1}{2} r^{2} \sin ^{2}(\chi-\phi)\right) \\
& \left.\quad \times \operatorname{erfc}\left(-\frac{1}{\sqrt{2}} r \cos (\chi-\phi)\right)\right] .
\end{aligned}
$$


At the centre of the core $r=0, P(\chi)=1 / 2 \pi$, i.e. the phase is completely undetermined. As $r$ increases, the distribution sharpens, and far from the core $P(c) \rightarrow \delta(\chi-\phi)$, reflecting the well-defined phase in this region where classical phase singularity $\psi=x+\mathrm{i} y$ dominates the field.

For vector waves whose state of classical polarization depends on position, an analogous argument would lead to the quantum smoothing of the polarization singularities through decay into modes with unrelated polarizations.

Analogous effects can be expected to smooth away the phase singularities in other types of wave. With sound, for example, the vortices are zeros of fluid pressure in the continuum description - the equivalent of classical optics. In the centres of these fleeting threads, the silence is destroyed by the faint pressure fluctuations of the gas moleculesfluctuations analogous to chaos of the quantum vacuum, audible in principle as Brownian fluctuations on a detector.

The radius $R_{\mathrm{S}}$ of this statistical core of an acoustic vortex, as detected by the human ear, for sound of intensity $d$ decibels, conventionally measured in terms of the faintest audible sound, can be estimated from two facts. The first is that the pressure amplitude of the Brownian fluctuations, calculated as (atmospheric pressure) $/ \sqrt{ } N$, where $N$ is the mean number of molecular impacts on the eardrum during the response time of the ear $\left((15 \mathrm{kHz})^{-1} \sim 7 \times 10^{-5} \mathrm{~s}\right)$, is, coincidentally or for reasons of evolution, comparable with that of the faintest audible sound. The second fact is that for omnidirectional random sound with wavelength $\lambda$ and mean pressure amplitude $p_{\mathrm{s}}$, the mean amplitude gradient at a nodal line (the analogue of (5)) is approximately $10 p_{\mathrm{s}} / \lambda$ (this is a simple consequence of results in [13]). It follows that

$$
R_{\mathrm{S}} \sim \frac{\lambda}{10^{1+d / 20}} .
$$

For a quiet musical note with loudness $40 \mathrm{~dB}, R_{\mathrm{s}} \sim 10^{-3} \lambda$ : for $500 \mathrm{~Hz}, R_{\mathrm{S}} \sim 0.6 \mathrm{~mm}$. We note that the ear is a broadband detector, unlike the atom in our proposal for detecting quantum cores of optical vortices, which is a tuned (that is, narrow-band) detector.

It is conceivable that quantum cores which are intrinsically richer (as opposed to ones that are merely more complicated) will emerge in situations other than that considered here. For example, we envisage expectation values involving higher powers of $\hat{E}(\mathbf{r})$, correlations between different values of $\mathbf{r}$ or different times, entanglements between the quantum states of the mode $N$ and the unoccupied modes, light confined (e.g. in a micromaser) so that all modes are discrete, and including the kinetic energy of the detecting atom.

We emphasize that the poverty of the core structure described here is associated with generic classical-optics phase singularities, that is, nodal lines in free space. In active media, by contrast, different core structures can occur, associated with nonlinearity. And it has recently been suggested [16, 17] that highly nontrivial new quantum phenomena, analogous to Hawking radiation, may be associated with more exotic logarithmic classical singularities such as the cores of classical vortices in rapidly rotating dielectric fluids.

According to the general philosophy outlined at the beginning of this paper, each deeper theory not only smooths the singularities of the previous, less general, theory that it supersedes, but contains the seeds of its own demise by introducing new singularities. This raises the enigmatic possibility that quantum-optical fields, considered 'in the wild' — that is, generically—might also possess singularities.

\section{Acknowledgments}

We thank Balazs Gyorffy for helpful conversations. MVB is supported by the Royal Society, and MRD is supported by the Leverhulme Trust.

\section{References}

[1] Nye J F 1999 Natural Focusing and Fine Structure of Light: Caustics and Wave Dislocations (Bristol: Institute of Physics Publishing)

[2] Airy G B 1838 On the intensity of light in the neighbourhood of a caustic Trans. Camb. Phil. Soc. 6 379-403

[3] Berry M V and Upstill C 1980 Catastrophe optics: morphologies of caustics and their diffraction patterns Prog. Opt. 18 257-346

[4] Berry M V 2001 Geometry of phase and polarization singularities, illustrated by edge diffraction and the tides Singular Optics 2000 (Alushta, Crimea) ed M S Soskin Proc. SPIE 4403 1-12

[5] Berry M V 1992 Rays, wavefronts and phase: a picture book of cusps Huygens' Principle 1690-1990; Theory and Applications ed H Bok and H A Ferwerda (Amsterdam: North-Holland) pp 97-111

[6] Berry M V 1998 Much ado about nothing: optical dislocation lines (phase singularities, zeros, vortices...) Singular Optics (Frunzenskoe, Crimea) ed M S Soskin and M V Vanetson Proc. SPIE 3487 1-5

[7] Nye J F and Berry M V 1974 Dislocations in wave trains Proc. R. Soc. A 336 165-90

[8] Berry M V 1981 Singularities in waves and rays Les Houches Lecture Series Session vol 35, ed R Balian, M Kléman and J-P Poirier (Amsterdam: North-Holland) pp 453-543

[9] Berry M V 2002 Coloured phase singularities New J. Phys. 4 66.1-14

[10] Berry M V 2002 Exploring the colours of dark light New J. Phys. 4 74.1-14

[11] Mandel L 1966 Antinormally ordered correlations and quantum counters Phys. Rev. 152 438-51

[12] Mandel L and Wolf E 1995 Optical Coherence and Quantum Optics (Cambridge: Cambridge University Press)

[13] Berry M V and Dennis M R 2000 Phase singularities in isotropic random waves Proc. R. Soc. A 456 2059-79

[14] Bethe H A and Salpeter E E 1977 Quantum Mechanics of One- and Two-Electron Atoms (New York: Plenum)

[15] Loudon R 2000 The Quantum Theory of Light (Oxford: Oxford University Press)

[16] Leonhardt U 2002 A laboratory analogue of the event horizon using slow light in an atomic medium Nature 415 406-9

[17] Leonhardt U 2002 Theory of a slow-light catastrophe Phys. Rev. A 65043818 